\title{
Individuation et individualisation
}

\section{Vincent Descombes}

\section{OpenEdition}

\section{Journals}

Édition électronique

URL : http://journals.openedition.org/ress/502

DOI : $10.4000 /$ ress.502

ISSN : 1663-4446

\section{Éditeur}

Librairie Droz

\section{Édition imprimée}

Date de publication : 1 décembre 2003

Pagination : 17-35

ISBN : 2-600-00912-4

ISSN : 0048-8046

Référence électronique

Vincent Descombes, «Individuation et individualisation », Revue européenne des sciences sociales [En ligne], XLI-127 | 2003, mis en ligne le 30 novembre 2009, consulté le 21 décembre 2020. URL : http:// journals.openedition.org/ress/502 ; DOI : https://doi.org/10.4000/ress.502 


\title{
Vincent DESCOMBES
}

\section{INDIVIDUATION ET INDIVIDUALISATION}

\section{LE PROBLÈME DE L'INDIVIDU}

\section{Qu'est-ce qu'une philosophie sociale?}

Je me propose de discuter un problème de philosophie sociale. Est-ce qu'une philosophie sociale est la même chose que ce qu'on appelle aussi une philosophie des sciences sociales? Cette dernière appellation peut s'entendre de deux façons, car il y a deux questions à poser à une science sociale quelconque: en quoi cette discipline est-elle une science? En quoi cette science est-elle sociale? La première de ces questions est méthodologique: en quoi ces disciplines qu'on réunit sous le nom de «sciences sociales» sont-elles précisément des sciences? La seconde de ces questions porte sur l'adjectif «social». Et, pour avoir une réponse à faire à cette dernière question, il faut d'abord accepter de la poser. Je réserverai l'appellation de philosophie sociale à la formulation et au traitement de la question portant sur le social comme tel. Nous pouvons ici nous munir d'un critère pour savoir si un penseur a développé une philosophie sociale. On considérera que quelqu'un a une telle philosophie s'il a pris au sérieux l'objection que fait Rousseau à la théorie politique des jusnaturalistes et de Hobbes, et s'il offre une réponse à cette objection. Dans le Discours sur l'origine de l'inégalité, Rousseau commence sa démonstration par une remarque bien connue, mais dont la portée n'est pas toujours aperçue:

\begin{abstract}
Les Philosophes qui ont examiné les fondements de la société, ont tous senti la nécessité de remonter jusqu'à l'état de Nature, mais aucun d'eux n'y est arrivé [...] Tous [...] ont transporté à l'état de Nature des idées qu'ils avaient prises dans la société; Ils parlaient de l'Homme Sauvage et ils peignaient l'Homme Civil' ${ }^{1}$.
\end{abstract}

Les «philosophes» que Rousseau vise ici sont identifiés dans la suite du texte par des allusions à leurs thèses. Il s'agit de Grotius, Locke, Pufendorf, Hobbes. Ces penseurs ont cherché à déterminer les fondements de la société. Par «fondements de la société », il ne faut pas comprendre les bases matérielles, mais les principes normatifs, c'est-à-dire des fondements qui justifient un ordre social, avec sa répartition des avantages et des charges. Rousseau observe que les philosophes en question font naître le gouvernement d'une transaction entre des

J.-J. Rousseau, Euvres complètes, Paris, Gallimard, Bibliothèque de la Pléiade, 1964, t. III, p. 132 . 
hommes naturels, «sans songer au temps qui dut s'écouler avant que le sens des mots d'autorité et de gouvernement pût exister parmi les Hommes » (ibid.).

L'objection de Rousseau est faite, comme on le voit sur le terrain du langage: comment les hommes naturels se comprennent-ils? Comment donnent-ils le même sens aux mots d'autorité et de gouvernement? On pourra dire aussi qu'elle est faite sur le terrain d'une philosophie de l'esprit: quelles idées pouvons-nous attribuer à des individus placés, par hypothèse, dans un contexte purement naturel (ce qui s'entend ici comme exclusion du lien social)?

Ainsi, Rousseau fait déjà l'objection qui sera celle de toute sociologie holiste à la conception individualiste de l'esprit qui est celle des théoriciens du droit naturel (lorsqu'ils veulent faire naître le droit de propriété et les autorités civiles d'un consentement des individus, donc d'une convergence de divers calculs individuels). D'après Rousseau, les philosophes qui ont parlé de l'homme à l'état de nature lui ont attribué des idées qui sont celles de l'homme social, «des idées qu'ils avaient prises dans la société».

La question de Rousseau doit donc être prise au sérieux contre Rousseau luimême, du moins si ce dernier prétend avoir fourni une autre explication constructiviste à la question qu'il avait posée. Je propose de tirer de cette réflexion de Rousseau une définition de la «philosophie sociale». On pourra demander: X (par exemple, Hegel, Nietzsche, Bergson, Husserl, Sartre, Wittgenstein) a-t-il une philosophie sociale? Cela voudra dire: trouve-t-on chez lui une réflexion sur la différence entre ce qui peut être fait ou pensé «dans l'état de nature» et ce qui ne peut être fait ou pensé que dans un «état de société »? La philosophie sociale permet de dire pourquoi et en quoi une activité est sociale. Elle détermine ce que c'est que la socialité (et elle peut être amenée à le faire contre le sens commun).

En vertu de cette définition de la philosophie sociale, il faut considérer que Durkheim ne propose pas seulement une explication sociologique de divers phénomènes, comme le suicide ou l'évolution des formes de vie religieuse, mais aussi une philosophie sociale. Il précise cette philosophie dans une note sur le sens du mot «société» destinée à figurer dans le Vocabulaire de la langue philosophique préparé par la Société française de philosophie et publié par André Lalande. Je reproduis cette définition:

\begin{abstract}
La grande différence entre les sociétés animales et les sociétés humaines est que, dans les premières, l'individu est gouverné exclusivement $d u$ dedans, par les instincts (sauf une faible part d'éducation individuelle, qui dépend elle-même de l'instinct); tandis que les sociétés humaines présentent un phénomène nouveau, d'une nature spéciale, qui consiste en ce que certaines manières d'agir sont imposées ou du moins proposées du dehors à l'individu et se surajoutent à sa nature propre: tel est le caractère des 'institutions' (au sens large du mot) que rend possible l'existence du langage, et dont le langage est luimême un exemple. Elles prennent corps dans des individus successifs sans que cette succession en détruise la continuité; leur présence est le caractère distinctif des sociétés humaines, et l'objet propre de la sociologie ${ }^{2}$.
\end{abstract}

L'homme à l'état de nature tel que le conçoit la théorie contractualiste du lien social ne peut disposer que de ses ressources individuelles («instincts » et «éduca-

\footnotetext{
Émile Durkheim, Textes, Paris, Minuit, 1975, t. I, p. 71.
} 
tion individuelle»). Puisque nous le supposons, par hypothèse, extérieur à toute société particulière, il ne possède pas les manières d'agir que Durkheim appelle institutions. On note que, dans cette intervention qui exprime sa position la plus réfléchie, le social ne se caractérise plus par le seul caractère contraignant ou obligatoire de certaines conduites, mais par leur caractère complexe. On ne peut pas rendre compte de ces conduites par les seules capacités naturelles d'un individu, car ces conduites supposent l'existence du langage qui est aussi l'exemple même d'une institution.

\section{Individualisation et socialisation selon Durkheim}

Dès le moment où la sociologie travaille à s'établir comme une discipline indépendante, et donc à spécifier l'objet propre de son enquête, elle rencontre une difficulté qui a un aspect intellectuel ou conceptuel et qui relève donc d'une philosophie sociale. Durkheim définissait ainsi l'objet de son étude sur La division $d u$ travail :

Quant à la question qui a été à l'origine de ce travail, c'est celle des rapports de la personnalité individuelle et de la solidarité sociale. Comment se fait-il que, tout en devenant plus autonome, l'individu dépende plus étroitement de la société ${ }^{3}$ ?

On connaît la réponse que donnait Durkheim. Il distinguait deux formes de la solidarité entre les parties dans le tout social: la «solidarité mécanique»est fondée sur la ressemblance des individus dans un groupe homogène, alors que la «solidarité organique » est fondée sur la complémentarité des fonctions spécialisées dans un groupe différencié. Pourtant l'énoncé même de la question comporte un point obscur: est-ce que le processus à étudier doit être caractérisé comme une individualisation croissante (progrès de l'autonomie) ou comme une socialisation croissante (progrès de la dépendance)? Puisque l'individu devient plus autonome, il semble y avoir émancipation à l'égard des solidarités sociales, donc individualisation. Mais puisque la solidarité devient organique, il semble qu'on doive conclure au contraire que les individus sont de plus en plus dépendants d'une organisation complexe.

La question de Durkheim est porteuse d'une contradiction interne, puisqu'on doit réunir deux caractères opposés dans une même qualification. Durkheim nous dit que l'individu était jadis plus indépendant (du fait de la simplicité morphologique du groupe), mais qu'il ne se représentait pas lui-même comme indépendant. Et qu'il est aujourd'hui plus dépendant (du fait de la complexité sociale), mais qu'il se représente lui-même comme un être autonome. Pourquoi ne se pensait-il pas comme indépendant au temps de la solidarité mécanique? C'est parce qu'il se pensait lui-même selon les exigences de la conscience collective. Comment peutil se penser aujourd'hui indépendant alors que son existence est liée aux autres par la solidarité organique? C'est parce que la division du travail a diminué l'emprise de la conscience collective (religion) sur le groupe et provoqué ce qu'on appelle chez les wébériens le «désenchantement du monde». Que penser de cette explication?

Émile Durkheim, La division du travail, Préface de la première édition, (1893), Paris, PUF, collection Quadrige, 1986, p. XLIII. 
Dira-t-on que l'individu relevant de la solidarité mécanique se croit plus dépendant qu'il ne l'est en réalité? Dira-t-on que l'individu moderne développe une «personnalité individuelle» et croit jouir d'une autonomie alors qu'il est en réalité plus dépendant des autres que ne l'était l'homme traditionnel?

En d'autres termes, comment se fait-il que les sociétés à solidarité mécanique croient jouir d'une unité organique, alors que les sociétés à solidarité organique se représentent leur unité comme un effet mécanique des initiatives individuelles?

\section{Le holisme de Durkheim}

Pour apercevoir la difficulté que pose à Durkheim l'emploi des notions d'individuel et de social, on peut se reporter à son compte rendu de l'ouvrage de Tönnies ${ }^{4}$. Il y a dans cette recension un paradoxe. Durkheim déclare approuver la distinction que fait Tönnies entre les deux formes de vie sociale (à savoir la «communauté», ou Gemeinschaft, et la «société», ou Gesellschaft). Il explique cette distinction en faisant appel à la relation du tout à ses parties. Oui, mais la façon dont il l'explique paraît ruiner le propos d'une sociologie holiste, c'est-àdire d'une discipline qui rend compte de la réalité de son objet (les faits sociaux) par les relations entre les parties au sein du tout ${ }^{5}$.

Considérons en effet l'explication par Durkheim de l'opposition entre les deux formes de vie sociale. Elle fait appel à la relation des parties au tout: dans la Gemeinschaft, le tout est donné avant les parties, alors que, dans la Gesellschaft, «ce sont maintenant les parties qui sont données avant le tout» (ibid., p. 387). Cette explication suggère qu'on doit pouvoir, dans la communauté, partir du tout (puisqu'il est donné préalablement) et découvrir, à partir d'une saisie du tout, la différenciation des parties, alors que c'est l'inverse lorsqu'on a affaire à la forme sociale de la Gesellschaft.

Pourtant, ce n'est pas à l'aide d'une analyse des rapports entre les parties au sein du tout que Durkheim explique le fonctionnement d'une Gemeinschaft. Il écrit en effet:

La Gemeinschaft, c'est la communauté. Ce qui la constitue, c'est une unité absolue qui exclut la distinction des parties. Un groupe qui mérite ce nom n'est pas une collection même organisée d'individus différents en relation les uns avec les autres; c'est une masse indistincte et compacte qui n'est capable que de mouvements d'ensemble, que ceux-ci soient dirigés par la masse elle-même ou par un des éléments chargés de la représenter. C'est un agrégat de consciences si fortement agglutinées qu'aucune ne peut se mouvoir indépendamment des autres [...] Le tout seul existe; seul il a une sphère d'action qui lui est propre. Les parties n'en ont pas.» (ibid., p. 384)

Ainsi, le tout qu'est la Gemeinschaft n'est pas véritablement un tout composé de parties distinctes, c'est une entité monolithique, un bloc qui ne peut agir qu'en masse. La définition que donne Durkheim de l'entité qu'est la société dotée d'une unité communautaire s'appliquerait tout aussi bien à une entité indivisible, à une

Compte rendu publié dans la Revue philosophique en 1889, donc peu avant La division du travail social, qui est de 1893. Voir E. Durkheim, Textes, op. cit., t. I, p. 383-390.

Dans ce qui suit, je ne retiendrai que les idées de Durkheim, sans m'occuper de savoir s'il a bien saisi la pensée de Tönnies. C'est en effet la difficulté inhérente à la philosophie sociale de Durkheim que je cherche à identifier et à éclaircir. 
unité qui compte pour un et un seul exemplaire dans le genre, bref à un individu. La Gemeinschaft, telle qu'elle vient d'être caractérisée, n'est pas du tout une totalité complexe, c'est l'équivalent d'un agent simple, c'est un individu collectif.

On voit les raisons qui conduisent Durkheim à concevoir le tout social comme un tout sans parties, donc comme une unité homogène, non différenciée. Si nous laissons les membres de la société se poser comme des individus distincts, si nous leur donnons une sphère d'action propre, nous risquons de ne plus avoir affaire à la Gemeinschaft, mais à une forme moderne de société contractuelle (dans laquelle les individus construisent le tout en établissant des relations entre eux).

Il n'en reste pas moins une difficulté de taille. Comment pouvons-nous parler d'un tout pour une entité qui se caractérise par le fait d'être sans parties? Si le concept de Gemeinschaft doit être compris ainsi, il ne répond pas à ce que nous attendons d'un concept holiste, c'est-à-dire de rendre possible l'analyse de la solidarité des parties au sein du tout.

Par quel prodige des individus peuvent-ils exister et agir comme s'ils n'avaient pas de sphère propre d'action, pas d'existence propre, mais seulement la capacité à participer à des mouvements d'ensemble? L'existence de la Gemeinschaft, qui est «le communisme porté à son plus haut point de perfection » (ibid.), repose sur le fait du «consensus ». Durkheim indique qu'il traduit par ce terme le mot Verständnis (qui peut signifier aussi qu'on s'entend dans le sens de se comprendre). Dans la socialité de type contractuel (Gesellschaft), l'accord ne peut être obtenu qu'au terme d'un processus de rapprochement des points de vue individuels, par la négociation ou la discussion. En revanche, dans la socialité de type communautaire (Gemeinschaft), cet accord est une harmonie donnée d'emblée, dans une heureuse communion des esprits qui réagissent de la même façon à l'événement. Durkheim décrit ainsi ce consensus:

\footnotetext{
C'est l'accord silencieux et spontané de plusieurs consciences qui sentent et pensent de même, qui sont ouvertes les unes aux autres, qui éprouvent en commun toutes leurs impressions, leurs joies comme leurs douleurs, qui, en un mot, vibrent à l'unisson [...] Pour que les consciences soient à ce point confondues, pour qu'elles participent ainsi à la vie les unes des autres, il faut qu'elles soient de même nature, ou qu'il y ait du moins entre elles de grandes ressemblances [...] (ibid., p. 384).
}

La condition du consensus (autre nom de la « conscience collective») est alors la fusion des «consciences individuelles» que rend possible le fait des ressemblances entre les expériences des uns et des autres (puisqu'ils n'ont pas des activités différenciées par la division du travail, mais au contraire des activités uniformes).

Ainsi, l'opposition des deux formes de vie sociale, du moins telle qu'elle est ici présentée par Durkheim, ne répond pas aux conditions d'une conception véritablement holistique du social. Selon son explication, la sociologie ne peut pas appréhender une totalité sociale à moins de réduire à rien l'activité des individus.

Au sein du groupe, il n'y a pas de mouvements, pas de changements dans la distribution des parties, puisqu'il n'y a pour ainsi dire pas de parties » (ibid., p. 386).

Mais, du même coup, le problème se pose de savoir pourquoi nous parlons d'un tout, donc d'une entité complexe dans laquelle on doit trouver de l'unité, mais aussi une diversité interne, et donc des rapports entre des parties. 
Or cette difficulté du texte reflète une autre obscurité de l'opposition conceptuelle qui nous est expliquée. Durkheim conclut sa recension du livre de Tönnies en expliquant qu'il approuve deux idées de l'auteur: d'abord, la distinction des deux formes sociales, ensuite la thèse selon laquelle la Gemeinschaft (société des statuts) précède partout la Gesellschaft (société du contrat). Mais Durkheim se sépare de Tönnies sur la conclusion à tirer de la constatation du «développement progressif de l'individualisme». D'après Tönnies, ce développement va rendre nécessaire une intervention de l'État pour maintenir un minimum de vie collective. Durkheim lui oppose que les deux formes de société ne sauraient être opposées à ce point: il s'agit, dans un cas comme dans l'autre, des formes que prend la vie sociale. Durkheim refuse donc d'apercevoir entre elles une « solution de continuité» (ibid., p. 390).

Durkheim a certainement raison sur un point: on ne peut pas, dit-il, commencer avec Aristote et finir avec Bentham. Il faut donc qu'il y ait quelque chose de commun aux deux formes. Oui, mais il y a deux façons de concevoir cette solution de continuité: on peut dire qu'il y a déjà de la Gesellschaft dans la Gemeinschaft, ou bien dire au contraire que le développement de l'individualisme ne va pas jusqu'à changer le tout qui précède ses parties en un tout artificiel construit par un accord contractuel entre les parties. Comment Durkheim luimême peut-il répondre?

Il ne semble pas Durkheim puisse expliquer comment un consensus, c'est-àdire une conscience collective, est possible dans une société de type contractuel, puisqu'il a lié le consensus à l'absence de cette complexité sociale qui rend possible la différenciation interne des fonctions et l'émergence des points de vue personnels. Ainsi, le seul consensus qu'on peut imaginer sera celui qui se dégage (laborieusement) au terme d'une confrontation des intérêts individuels et des opinions personnelles. Mais cela veut dire qu'un groupe dont la forme sociale a, de façon prédominante, les traits de la Gesellschaft ne relève plus d'une sociologie holiste. Si l'on devait accepter cette thèse, ce serait la faillite du programme sociologique de Durkheim.

Ainsi, notre difficulté est la suivante: si nous prenons au mot ce que la société moderne nous dit d'elle-même, elle repose sur des principes entièrement opposés aux sociétés qui se pensent sur un mode de type gemeinschaftlich. Il n'existerait donc un point de vue universel qui réunirait sous des principes communs les sociétés traditionnelles et la société moderne. Il ne serait pas concevable de réunir l'étude des unes et des autres dans une seule discipline d'anthropologie sociale.

La difficulté que nous avons rencontrée peut se formuler comme le problème sociologique de l'individu: comment concevoir la «sphère d'action » propre à un agent individuel dans les conditions d'une «solidarité mécanique» ou, si l'on veut, d'une société traditionnelle? Or nous sommes en train d'apercevoir que, pour poser ce problème, il faut affronter aussi ce qu'on peut appeler le problème anthropologique de l'individu, c'est-à-dire le problème de notre usage du concept d'individu dans le cadre d'une sociologie qui se veut comparative.

\section{Le problème anthropologique de l'individu}

Il s'agit donc de se demander, de façon générale, comment il y a déjà des individus dans une société traditionnelle (à solidarité mécanique) et comment il y a 
encore une vie sociale dans une société moderne (à solidarité organique). La question est de savoir si la sociologie doit avoir une ambition anthropologique, c'està-dire viser à saisir ce qu'il y a de commun à l'homme traditionnel et à l'homme moderne. En principe, il y a quatre réponses possibles à notre problème. Donnons le nom de «société durkheimienne» à un groupe qui apparaît doté d'une conscience collective, dans le sens de «représentations collectives» fournissant les fondements d'un consensus au regard duquel les conduites non conformes sont jugées incorrectes, fautives, répréhensibles, etc. Les quatre possibilités sont alors:

(I) Toutes les sociétés humaines sont des sociétés durkheimiennes, c'est-à-dire des totalités dotées d'une conscience collective. (Cette réponse représente le point de vue d'une sociologie conforme au programme fondateur de Durkheim.)

(II) Il a existé des sociétés durkheimiennes, mais il n'y en a plus, du moins dans notre partie du monde, car nos propres formes de vie sociale ne reposent pas sur la conscience collective, mais au contraire sur le pluralisme: l'exigence du consensus tend à devenir purement «procédurale». (L'histoire a produit le déclin de la Gemeinschaft au profit d'une pure Gesellschaft. Les théories sociologiques de Durkheim valent pour les religions tribales et les sociétés primitives, mais pas pour nous. On note que cette position était souvent celle des philosophes contemporains de Durkheim.) ${ }^{6}$

(III) Il existe encore des sociétés durkheimiennes, mais cela ne sera plus le cas après l'abolition de ce qui reste archaïque ou aliénant dans l'organisation sociale. (Cette réponse est une variante de la précédente: elle prend acte, comme la précédente, de la transition à une socialité moderne, mais estime que nos propres formes d'organisation reposent encore beaucoup trop sur de vieux préjugés.)

(IV) Il n'existe pas et il n'a jamais existé de sociétés (de type durkheimien).

Traduites en thèses sur la discipline sociologique, ces propositions donnent ceci :

(I) Une sociologie holiste a un objet partout où il y a une présence humaine.

(II) Une sociologie holiste a eu un objet, mais elle n'en a plus à l'âge moderne.

(III) Une sociologie holiste a encore un objet (survivances, archaïsmes), mais il est concevable (et d'ailleurs souhaitable) qu'elle n'en ait plus.

(IV) Une sociologie holiste n'a jamais eu d'objet.

Je chercherai à préciser l'origine des obscurités qui nous arrêtent chez Durkheim en partant d'un commentaire que fait Raymond Boudon, et qui me semble avoir le sens suivant: il est faux de prétendre que Durkheim a soutenu (I), autrement dit qu'il soit le fondateur d'une sociologie holiste, car il lui arrive de soutenir la thèse (II); or cette thèse (II) est instable; à la réflexion, elle se change facilement en thèse (IV), laquelle correspond à l'individualisme méthodologique.

6 $\quad$ Voir Durkheim, op. cit., t. I, p. 57. 
Boudon souligne que, pour Durkheim, l'individualisme n'est pas propre à l'époque moderne. Il se réfère à plusieurs reprises à une remarque de Durkheim: «l'individualisme est un phénomène qui ne commence nulle part» ${ }^{7}$. Par cette référence, Boudon veut opposer Durkheim lui-même aux sociologues qui font de l'individualisme un trait distinctif des sociétés modernes (je veux dire, un trait distinctif des valeurs de ces sociétés). Pour lui, ce recours à des différences culturelles de société à société présente deux inconvénients. D'abord, on invoque à des fins explicatives des forces culturelles «cachées » responsables des phénomènes, forces qui en réalité n'expliquent rien du tout. Ensuite, on est conduit à des conclusions fâcheuses comme celle que Boudon attribue à S. Huntington (les civilisations sont «des totalités incapables d'échapper à leur unicité et à leur destin » ${ }^{8}$, le conflit entre elles est donc au fond inévitable).

Nous remarquons que le mot «totalité » fait son apparition. Pour l'individualiste, il s'agit bien de s'opposer à une position holiste. Toutefois, on peut s'interroger: les totalités dont on nous parle sont-elles à concevoir comme des masses agissant en bloc ou comme des systèmes complexes dotés d'une différenciation interne? D'autre part, s'agit-il d'introduire des «forces culturelles»? Ne s'agit-il pas bien plutôt d'introduire des institutions «au sens large du mot», sens que Durkheim précisait en parlant des manières d'agir pour les membres d'un groupe (et non des facteurs invisibles agissant à leur insu sur leur conduite)? Est-ce la culture qui agit ou est-ce l'agent particulier? Si le social est à chercher dans la manière d'agir d'un agent, il va de soi que l'action doit être imputée à cet agent, pas à des «forces » qui opéreraient sur l'individu à son insu.

Se réclamant de Durkheim - l'individualisme a toujours existé - Boudon juge que c'est une erreur d'opposer des cultures qui reconnaissent l'individu et d'autres qui ne le font pas. Par exemple, écrit-il, c'est une erreur de dire qu'une culture villageoise qui obéit à la règle de l'unanimité (plutôt qu'à celle du vote à la majorité) le fasse parce qu'elle ne reconnaît pas l'individu, seulement l'opinion du groupe. On croit expliquer le palabre en invoquant la culture, c'est-à-dire une «force culturelle» qui produit l'opinion des individus: «là où les sociétés modernes seraient individualistes, les sociétés villageoises seraient unanimistes » (ibid., p. 36). De sorte que les membres du village, soumis à «l'action de cette force culturelle» qu'est la valorisation du rapport au groupe, se comporteraient comme les citoyens de la cité de Rousseau et n'attacheraient pas d'importance aux intérêts personnels. En fait, objecte Boudon, la règle de l'unanimité donne à tous les individus un pouvoir de veto qu'ils n'ont pas dans une démocratie fonctionnant au vote majoritaire. La procédure du palabre, dans ces sociétés villageoises, ne témoigne pas d'un mépris de l'individualité, elle est pleine d'un sens individualiste dans les conditions de vie d'un village. «Il est donc illusoire de croire qu'il existe des sociétés où l'individu se percevrait comme dissous dans le groupe» (ibid.).

Est-ce que Boudon a prouvé que Durkheim était un individualiste méthodologique sans le savoir? Non, sans doute, mais je crois néanmoins qu'il a fort bien vu la faille dans la position, officiellement holiste, du fondateur de l'école française

Cité dans: Raymond Boudon, Raison, bonnes raisons, Paris, PUF, 2003, p. 14, p. 36.

$8 \quad$ R. Boudon, ibid., p. 14. 
de sociologie. Impossible de nier qu'il y a une contradiction entre la thèse holiste de Durkheim sur la conscience collective et sa thèse sur la continuité du processus d'individualisation. D'une part, Durkheim veut opposer deux types de société, et il définit le type traditionnel ou «communautaire» par l'absence d'un individu indépendant. D'autre part, Durkheim veut que le processus de l'individualisation soit continu. Les deux thèses sont incompatibles. Il apparaît donc justifié de remarquer que si Durkheim veut maintenir cette dernière thèse (anthropologique), il doit renoncer à parler d'une forme sociale dans laquelle l'individu n'existait pas et il doit par conséquent embrasser les vues de l'individualisme méthodologique.

Durkheim éprouve le besoin de poser sa thèse de la continuité du processus d'individualisation parce qu'il veut écarter une interprétation superficielle du phénomène qui en ferait un événement récent, peut-être éphémère. En réalité, le processus de l'émancipation individuelle est irréversible. L'histoire universelle montre que le «type collectif» d'homme tend à décliner au profit de l'individualité. S'il s'agissait d'une tendance récente (produit de notre civilisation) ou d'un «événement unique dans l'histoire des sociétés», on pourrait se demander si ce n'est pas réversible. En fait, l'évolution se fait dans ce sens « depuis les temps les plus lointains ».

L'individualisme, la libre pensée ne datent ni de nos jours, ni de 1789, ni de la réforme, ni de la scolastique, ni de la chute du polythéisme gréco-latin ou des théocraties orientales. C'est un phénomène qui ne commence nulle part, mais qui se développe sans s'arrêter, tout le long de l'histoire'.

C'est un phénomène qui ne commence nulle part. Dans un texte qui précède de peu celui qui vient d'être cité, ce phénomène est décrit, très classiquement, comme un processus de sécularisation. Durkheim croit pouvoir observer ceci: le déclin de l'emprise du religieux sur le social a pour contrepartie le progrès de l'individualisme, c'est-à-dire le fait que l'homme individuel devient de plus en plus actif, de plus en plus un «sujet» au sens d'un agent autonome.

\begin{abstract}
Or s'il est une vérité que l'histoire a mise hors de doute, c'est que la religion embrasse une portion de plus en plus petite de la vie sociale. A l'origine, elle s'étend à tout; tout ce qui est social est religieux. Puis, peu à peu, les fonctions politiques, économiques, scientifiques s'affranchissent de la fonction religieuse, se constituent à part et prennent un caractère temporel de plus en plus accusé. Dieu [...] abandonne le monde aux hommes et à leurs disputes [...] L'individu se sent donc, il est réellement moins agi ; il devient davantage une source d'activité spontanée [...] Cette régression n'a pas commencé à tel ou tel moment de l'histoire: mais on peut en suivre les phases depuis les origines de l'évolution sociale (ibid., p. 143-144).
\end{abstract}

D'après Durkheim, l'homme devient de plus en plus «une source d'activité spontanée». Auparavant, il ne l'était pas, mais pour des raisons qui, indique-t-il, tiennent à la façon dont il se représentait sa propre réalité: il y a donc passage d'un état aberrant à un état normal ou raisonnable. Ce portrait de l'homme primitif repose sur un postulat délicat: l'homme ne se pensait pas comme un individu, donc il n'était pas un individu. Nous retrouvons ici la difficulté notée en commençant: qu'est-ce qui importe? Est-ce le fait que l'homme moderne soit plus solidaire (de fait) ou est-ce le fait qu'il se représente lui-même comme étant autonome?

9 Émile Durkheim, De la division du travail social, op. cit., p. 146. 
Le schéma durkheimien est certes anthropologique, mais n'est-ce pas au prix d'une identification de la modernité avec la rationalité au sens normatif? La modernité n'est plus seulement une époque historique, c'est l'époque dont les idées majeures sont satisfaisantes pour l'esprit, à la différence des autres époques dont les idées étaient au fond aberrantes. Ainsi, l'homme moderne sait qu'il est une source d'activité, alors que l'homme traditionnel était une source d'activité, mais ne voulait pas le savoir. Un tel schéma n'est pas conforme au principe comparatif. Il manque ici un moyen de concilier deux propositions incontestables:

$1^{\circ}$ Dans toute société humaine, on rencontrera des individus humains au sens où Durkheim définit l'individu comme «une source d'activité spontanée».

$2^{\circ}$ L'avènement des idées modernes sur l'individualité ou sur la «personnalité humaine» suppose une rupture nette dans les valeurs et les idéaux qui définissent la «conscience collective» de la société.

Je crois que c'est précisément ce moyen que nous a donné Louis Dumont en distinguant un sens empirique du mot «individu» et un sens normatif. Dumont a voulu lever une confusion qu'il apercevait dans la pensée des fondateurs de la discipline sociologique. La confusion provient de ce que le sociologue n'a pas réussi à s'émanciper du sens commun de son milieu d'origine. On se figure que, si une société ne partage pas nos valeurs individualistes, c'est qu'elle est d'une façon ou d'une autre incapable de faire des distinctions que nous savons faire. Elle ne sait pas imputer des actions à des sources individuelles d'activité. Inversement, si elle sait procéder à de telles imputations individuelles, c'est que l'individualisme est déjà en marche.

Dumont explique qu'en parlant d'individu, le sociologue peut viser l'une des deux choses suivantes:

1. L'agent empirique, présent dans toute société, qui est à ce titre la matière première principale de toute sociologie.

2. L'être de raison, le sujet normatif des institutions; ceci nous est propre, comme en font foi les valeurs d'égalité et de liberté, c'est une représentation idéelle et idéale que nous avons ${ }^{10}$.

Il y a un sens où l'individu existe partout: non seulement dans notre société, mais dans toute société humaine. C'est l'individu au sens de l'individuation. Et il y a un autre sens dans lequel l'individu comme tel n'existe nulle part, ce qui veut dire que la réalité n'est jamais entièrement adéquate à l'exigence d'autonomie que porte l'idée normative d'individualité. Il s'agit alors de l'individu au sens de l'individualisation.

Qu'est-ce qui nous permet de dire que l'individu comme être autonome, donc donné à lui-même préalablement à toute socialité, est une «représentation idéale», pas une réalité empirique? La réponse relève en fin de compte, selon Dumont, d'une vue sur le fonctionnement de l'esprit humain. Sans doute, l'homme moderne se représente lui-même comme un individu indépendant, au

10 Louis Dumont, Homo hierarchicus, Paris, Gallimard, 1966, p. 22. 
moins lorsqu'il pense. Mais en réalité il n'en est pas moins un être social que l'homme traditionnel, et c'est là ce qui rend possible la comparaison anthropologique entre des formes d'humanité qui sont séparées par une révolution des valeurs, donc par une discontinuité historique.

Là-dessus il suffit d'observer que les hommes concrets ne se comportent pas: ils agissent
avec une idée en tête, fût-elle de se conformer à l'usage. L'homme agit en fonction de ce
qu'il pense, et s'il a jusqu'à un certain point la faculté d'agencer ses pensées à sa guise,
de construire des catégories nouvelles, il le fait à partir des catégories qui sont sociale-
ment données, comme leur liaison avec le langage suffirait à le rappeler. (Ibid., p.19).

L'homme agit: c'est un individu qui est l'auteur de son action. Il agit en fonction de projets et d'intentions qu'il forme: c'est un individu qui a tel ou tel projet. Dans l'un et l'autre cas, nous parlons de l'individu au sens de l'individuation: dire qu'il y a eu telle ou telle action, c'est dire qu'une personne, ou deux personnes, ou plus, ont fait cette action. Dire qu'un projet existe, c'est dire que quelqu'un a ce projet. Comment distinguer en effet une action d'une autre action? Il faut passer par l'individuation de l'agent.

Mais les catégories qui lui servent à «agencer ses pensées» (à former des projets, à délibérer) sont sociales. Autrement dit, l'homme qui agit en fonction de ce qu'il pense est un homme social, puisque l'agencement de ses pensées ne résulte pas d'une architecture dont il serait l'auteur, mais d'une application de catégories qui sont empruntées à un groupe social. Par conséquent, l'idée moderne de l'individu, au sens normatif, correspond à un projet d'individualisation dans l'agencement des pensées. Le progrès de l'individualisme ne saurait consister à individuer des êtres qui n'auraient été jusque là que des parties virtuelles fondues dans la masse indistincte d'un groupe agissant comme un seul homme. L'individu qui est en cause dans les valeurs individualistes, ce n'est pas l'individu au sens descriptif de l'individuation, c'est l'individu au sens normatif de l'individualisation. Individualiser ne veut pas dire ici reconnaître à l'individu une «sphère d'action » propre, au sens d'une opposition entre une action individuelle et une participation à l'action collective, comme le donnait à penser Durkheim dans son explication de l'opposition entre la Gemeinschaft et la Gesellschaft. Il s'agit plutôt de lui reconnaître un domaine privé, une sphère d'autonomie, en ce sens que c'est à lui, s'il le veut et s'il le peut, de fixer les principes et les règles de sa conduite dans ce domaine qui lui est réservé.

La distinction que propose Dumont vise chez lui à surmonter la difficulté rencontrée dans les positions de Durkheim que j'ai rappelées. Je crois pouvoir confirmer la nécessité de distinguer ces deux sens en discutant directement la formulation de la position (IV), celle de l'individualisme méthodologique, dans le livre récent de Boudon.

\section{LE POSTULAT INDIVIDUALISTE}

\section{Boudon formule ainsi ce qu'il appelle le «postulat» de l'individualisme:}

Le premier postulat [...] veut que tout phénomène social soit le produit d'actions, de décisions, d'attitudes, de comportements, de croyances, etc. individuels, les individus étant les seuls substrats possibles de l'action, de la décision, etc., dès lors que l'on prend ces 
notions dans un sens non métaphorique [...] Ce premier postulat a un fondement ontologique: il déclare que seuls individus humains peuvent être le siège de croyances, de désirs, d'intentions [...] (Raison, bonnes raisons, op. cit., p. 19).

On peut comprendre que la philosophie sociale de l'individualisme repose sur deux propositions, la première formulant une évidence, donc un axiome ontologique, la seconde énonçant une conséquence qui est le postulat individualiste proprement dit.

L'axiome pose que le sujet des verbes d'acte mental ou d'action intentionnelle ne peut être qu'un agent personnel individuel. C'est ce que veut illustrer l'adage par lequel Boudon illustre ensuite ce point: le Parti ne pense pas, seuls les membres du Parti pensent.

Le postulat individualiste énonce alors que les phénomènes sociaux, puisqu'ils consistent dans des actions et des pensées qui ont pour sujets (dans le sens de substrats) des individus humains, sont eux-mêmes des phénomènes de composition, des résultantes.

Cette explication du postulat proprement dit par l'axiome «ontologique» est manifestement insatisfaisante. En effet, le postulat est censé représenter une prise de parti en faveur d'une position théorique substantielle: il consiste à exclure une autre position théorique, celle qui est qualifiée de «holisme» (ibid., p. 25) et qui veut que les phénomènes sociaux soient irréductibles à de simples effets de composition. Pourtant, l'axiome sur lequel l'auteur veut appuyer son postulat n'est pas un principe théorique substantiel, c'est le simple rappel d'une condition d'intelligibilité, une «remarque grammaticale», comme dirait Wittgenstein.

L'axiome déclare qu'il ne peut pas y avoir de décision sans un décideur, d'action sans un agent, d'attitude sans un sujet de cette attitude. Nous reconnaissons le vénérable axiome scolastique: actiones sunt suppositorum. C'est un principe qu'on peut qualifier de «grammatical» parce qu'il fixe les conditions de sens de la construction d'un verbe d'action (et pas seulement d'action intelligente): on ne peut utiliser un verbe d'action pour imputer une action à un agent que si l'agent qu'on mentionne est individué, s'il peut être identifié comme étant tel ou tel agent.

Soit, par exemple, une action à laquelle nous assistons et que nous identifions comme une partie d'échecs. Nous ne pouvons pas juger qu'une partie d'échecs est en train de se jouer s'il n'y a pas deux joueurs en train de jouer l'un contre l'autre aux échecs. Pour qu'il existe une partie d'échecs, il faut donc qu'on puisse désigner deux individus comme étant les adversaires de cette partie d'échecs. Ainsi compris, l'axiome est complètement anodin pour la philosophie sociale, car il ne s'occupe absolument pas de la différence entre des conduites purement individuelles et des conduites sociales: entre la conduite d'un individu dont le sens repose sur un agencement d'idées purement individuelles (qu'aurait pu avoir l'Homme Naturel) et celle d'un individu dont le sens repose sur un agencement d'idées prises dans la société.

Que les actions des individus - qu'elles soient coordonnées ou non, et qu'elles le soient par des calculs individuels ou autrement - aient pour agents des individus, c'est là une tautologie. L'axiome ne nous dit nullement si nous pouvons rendre compte entièrement de la manière d'agir par un calcul individuel des raisons que l'individu peut fournir par lui-même, ou s'il faut renvoyer, par delà le raisonnement dont chaque individu est capable, à des modèles d'action et de 
penser qui procurent à notre agent individuel la règle à suivre dans son action pour se montrer «rationnel», pour «agir au mieux», pour «faire ce qu'il convient de faire».

En revanche, le postulat de l'individualisme méthodologique n'est pas un simple rappel de la grammaire des verbes psychologiques. C'est une thèse substantielle puisqu'il pose qu'il n'y a rien de plus, dans une action sociale (par exemple, deux personnes qui jouent aux échecs) que la composition de deux actions individuelles. On pourrait exprimer la différence entre le sens trivial de l'axiome et le sens réducteur du postulat en demandant à l'individualiste de choisir entre les deux thèses ontologiques suivantes sur ce qui existe chaque fois qu'il se joue une partie d'échecs:

(I) Il n'y a rien de plus, dans la catégorie des substrats (des suppôts), que les deux joueurs d'échecs.

(II) Il n'y a rien de plus, dans aucune catégorie ontologique, que, d'une part, les deux joueurs (dans la catégorie des agents identifiables), et, d'autre part, les actions individuelles de l'un et de l'autre (dans la catégorie des événements).

Selon la thèse (I), il n'y a pas à l'œuvre, en plus des deux joueurs individuels, un autre agent qui serait une entité collective, par exemple l'«âme du jeu» ou la communauté des joueurs. On revient à l'évidence de l'adage «Le Parti ne pense pas ». Personne ne pourrait expliquer qu'il faut être trois pour jouer aux échecs. La thèse (I) est donc que si deux personnes jouent ensemble, c'est qu'il y a deux joueurs.

Selon la thèse (II), il suffit de deux catégories ontologiques pour rendre compte de l'existence d'une partie d'échecs. A cela, je crois qu'on peut répondre sans hésiter que c'est faux, que nous nous ne souhaitons pas borner ainsi notre table des catégories. Considérons en effet les catégories nécessaires du point de la syntaxe d'une description d'une partie d'échecs. On a besoin de la catégorie des noms propres, pour désigner les joueurs, et de la catégorie des verbes d'action, pour identifier leurs gestes. Ces catégories syntaxiques correspondent aux suppôts et à leurs actions. Mais il faut aussi la catégorie syntaxique des adverbes, pour dire comment les agents agissent et en quoi leurs gestes sont des coups dans une interaction définie par certaines règles, celles du jeu des échecs. Sinon, on ne pourrait pas faire la différence entre deux personnes qui jouent aux échecs et deux personnes qui reproduisent les gestes du jeu d'échecs, mais sans jouer aux échecs.

Ce dernier point appelle une réflexion sur les règles. J'ai cité en commençant un texte où Durkheim posait ce qui distinguait selon lui une société humaine d'une société animale: les membres d'une telle société manifestent la socialité qui est la leur par la manière dont ils agissent. Leurs actions répondent à des modèles institutionnels, dans un sens large où les règles du jeu d'échecs, tout comme les règles du langage, sont des institutions. Que l'agent soit individuel, et que son action ait dont le caractère d'une action imputable à un agent individuel, ne nous dit pas si la manière dont cet agent agit manifeste une régulation naturelle (instinct) ou une signification sociale (institution).

Je dois maintenant montrer comment nous ne pouvons pas nous dispenser d'une troisième catégorie correspondant aux «manières d'agir», de façon à pouvoir faire la différence entre une conduite régulière parce que contrôlée, «de 
l'intérieur», par un mécanisme naturel, et une conduite régulière parce que gouvernée, «de l'extérieur», par une norme sociale. Bien entendu, cette norme n'est extérieure qu'à l'individualité naturelle de l'individu. Elle ne doit pas être conçue comme un «facteur extérieur », une «force cachée» par laquelle une entité occulte, la culture, agirait sur les individus humains. La culture, le social, ou, si l'on veut, l'ensemble des normes ne sont nullement des entités agissantes. Pour tirer au clair ce dernier point, je m'appuierai sur les analyses de Wittgenstein.

\section{UNE PHILOSOPHIE SOCIALE DE L'ESPRIT}

\section{Wittgenstein a-t-il une philosophie sociale?}

Que vient faire Wittgenstein dans notre débat? A-t-il jamais porté le moindre intérêt à la science sociale, si l'on excepte les quelques remarques sur Le rameau d'or de Frazer? J'ai introduit en commençant un critère permettant de dire si un penseur a développé une philosophie sociale: avoir une philosophie sociale, c'est avoir mené une réflexion sur la différence entre ce qui peut être fait «dans l'état de nature » et ce qui ne peut être fait que dans un «état de société». La philosophie sociale permet de dire pourquoi et en quoi une activité est sociale: une activité est sociale si elle suppose que l'agent ait pris ses idées «dans la société».

Il se trouve que Wittgenstein a posé une question de ce genre à propos des activités de type linguistique. Il a comparé le fait de se servir d'un mot au fait de se servir de pièces au jeu d'échecs. Dans les deux cas, la compétence de l'individu consiste à savoir obéir à une règle dans sa conduite. De telles activités, a-t-il expliqué, ne sont possibles que sur le fond d'un consensus. Ces activités sont donc sociales, ce qui veut dire qu'elles exigent plusieurs agents, même en l'absence d'une coordination visible ou actuelle des mouvements de plusieurs sociétaires. Toutefois, ce consensus ne requiert aucunement une fusion des consciences individuelles dans une seule expérience commune, et encore moins la fusion des agents individuels dans un seul agent collectif.

En vertu de ce critère qui définit le social par le consensus, on doit compter comme activités sociales non seulement ce que notre sens commun place dans cette catégorie (les actions faites à plusieurs, les actions coordonnées), mais certaines activités solitaires. Il y a - telle est la vue dérangeante qui est au fond de l'argument de Wittgenstein - des activités qui peuvent être exercées par un agent individuel sans la coopération de quelqu'un d'autre, mais qui ne peuvent pas être identifiées en dehors d'un contexte social. Si elles ne peuvent pas être identifiées par les autres que l'agent en dehors de ce contexte, elles ne peuvent pas non plus l'être par l'agent lui-même, ce qui veut dire qu'il ne peut lui-même penser son action qu'en vertu du contexte social que lui a procuré son apprentissage des règles appliquées dans ses opérations solitaires.

\section{Activités naturelles et activités sociales}

Wittgenstein fait lui-même la comparaison entre les actions manifestement contextuelles et les actions dont le caractère contextuel est loin d'être évident. Dans un passage célèbre des Remarques sur les fondements des mathématiques (VI, § 45), il nous demande de comparer ces deux questions: «Puis-je faire du 
commerce tout seul?», «Puis-je calculer tout seul?». La première activité est évidemment sociale: Robinson sur son île ne peut pas ouvrir une boutique. $\mathrm{Ou}$, plutôt, il peut faire les gestes d'un marchand qui réunit des denrées et les présente à un acheteur éventuel. Puisqu'il se sait seul sur l'île, ses gestes ne peuvent pas avoir le sens d'entreprendre de faire du commerce. La seconde activité, à première vue, n'est pas du tout sociale. Robinson peut très bien faire des calculs, il n'a pas besoin pour cela d'un partenaire.

La démonstration de Wittgenstein passe, pourrait-on dire, par deux étapes. La première étape consiste à considérer une activité ouvertement contextuelle et à noter ce qui la rend contextuelle. La seconde étape consiste à considérer un cas intermédiaire entre l'activité ouvertement contextuelle et celle qui, nous semblet-il d'abord, ne l'est pas du tout.

Il donne comme un exemple d'activité dont le sens dépend du contexte: une cérémonie de couronnement royal ${ }^{11}$. Dans le contexte britannique, les divers éléments de la cérémonie (couronne d'or, manteau de pourpre) sont les symboles mêmes du faste et de la dignité. Mais, si nous déplaçons ce matériel dans le contexte d'un autre pays où l'or, matériau abondant, est jugé vulgaire, où la couronne fait l'effet d'une coiffure ridicule, toute la cérémonie change de sens. Ainsi, l'identité matérielle des gestes et des instruments ne suffit pas à fixer le sens de l'action sociale, car ce sens est fonction du milieu environnant (Umgebung).

En revanche, faire un calcul arithmétique est une opération qu'un individu peut faire alors qu'il est tout seul. On pourrait donc penser que ce n'est pas contextuel. Ou bien encore, on pourrait penser que c'est contextuel, mais que le contexte est celui d'une biographie individuelle, pas d'une histoire commune. Il paraît difficile de parler d'une activité sociale. Et, si ce n'est pas socialement contextuel, on peut imaginer que quelqu'un le fasse pour la première fois dans l'histoire de l'humanité, sans modèle, ce qui veut dire qu'il le ferait sans en prendre l'idée «dans la société». L'Homme Naturel pourrait le faire.

La stratégie de Wittgenstein est de discuter divers exemples qui forment un troisième cas, intermédiaire entre l'activité de coopération et l'activité de suivre individuellement une règle. Cette discussion permet de passer de ce que personne ne conteste - la socialité d'une cérémonie traditionnelle - à quelque chose qui est beaucoup moins clair et que beaucoup jugeront «contraire à nos intuitions »: la socialité d'une activité réglée de type solitaire.

Selon le concept banal du social, une partie de jeu d'échecs est quelque chose de social parce qu'il faut être deux pour jouer, et que les joueurs doivent se mettre d'accord (quelles règles, qui commence, etc.). Or une partie d'échecs est également une affaire sociale dans un autre sens, moins trivial. Elle l'est en vertu de sa forme, en vertu de ce qui la constitue comme partie d'échecs: les épisodes qui constituent (diachroniquement) la partie d'échecs sont constitués comme une suite de coups dans le jeu par le fait d'être des mouvements accomplis selon les règles. Et cette forme du comportement est sociale. Wittgenstein nous demande d'imaginer la situation suivante. Nous sommes des ethnographes de terrain, et nous observons ce que font des gens dans le village qui est l'objet de notre enquête:

${ }^{11}$ Wittgenstein, Recherches philosophiques, $\$ 584$. 


\begin{abstract}
Il est bien sûr concevable que, dans un peuple qui ne connaît pas le jeu d'échecs, deux personnes soient assises devant un échiquier et qu'elles fassent les coups d'une partie d'échecs - et cela avec en outre tous les phénomènes mentaux concomitants (mit allen seelischen Begleitererscheinungen). Et si nous observions cela, nous dirions qu'elles jouent aux échecs. (Recherches philosophiques, § 200):
\end{abstract}

Nous le dirions sans hésiter puisque nous aurions devant nous toutes les apparences d'une partie d'échecs. Et, pourtant, nous aurions tort de le dire puisqu'il manquerait le contexte d'une pratique instituée du jeu d'échecs. Sans ce contexte, les individus peuvent singer une partie d'échecs, mais pas jouer aux échecs. Il en est ainsi, non pour des raisons psychologiques, mais pour des raisons logiques: le prédicat «jouer aux échecs» ne peut pas s'appliquer à des individus sur la seule base de leurs gestes et des données mentales qui se présentent à leurs consciences. Seul peut être un joueur d'échecs quelqu'un qui participe à une pratique bien établie du jeu d'échecs.

\title{
3. La réduction individualiste est-elle possible?
}

On pourrait dire que l'individualisme méthodologique s'appuie sur notre première réaction, laquelle ne tient pas compte du contexte. L'argument individualiste serait ici le suivant. D'abord, il concéderait que jouer aux échecs est une activité sociale, tout comme faire du commerce ou se marier sont des activités sociales. Il faut être deux pour jouer aux échecs. Mais, ensuite, il contesterait que cette socialité soit irréductible. Il ferait remarquer qu'une partie d'échecs est le produit de la rencontre de deux intentions individuelles en cours d'exécution. La socialité du jeu d'échecs doit s'analyser en une combinaison intersubjective de deux activités mentales individuelles (chacun désirant jouer aux échecs, et désirant le faire avec l'autre partenaire).

Dans une situation comme celle des deux villageois qu'imagine Wittgenstein, rien ne nous empêche - dira l'individualiste méthodologique - de supposer que les deux individus ont les mêmes états d'esprit que des joueurs d'échecs. Chacun, de son côté, a l'intention de jouer à un jeu. Ils ont cette intention ensemble, mais chacun possède son intention indépendamment de l'autre. Le fait que le jeu ne soit pas présent dans la culture de leur groupe n'a pas d'importance. Nous pourrions dire que, dans le situation donnée en exemple, les deux individus viennent d'inventer par eux-mêmes (sous nos yeux) le jeu d'échecs. L'aventure est certes improbable, mais elle n'est pas logiquement exclue.

Que signifie ce raisonnement du point de vue d'une philosophie de l'esprit? Il suppose qu'on puisse tenir une intention, quelle qu'elle soit, comme un état strictement individuel J'ai besoin d'un partenaire (et d'objets servant de pièces et d'échiquier) pour jouer aux échecs. Je n'en ai pas besoin pour avoir l'intention (Absicht) de jouer aux échecs. Je peux avoir cette intention en l'absence de tout partenaire. Mieux, je peux l'avoir quoi qu'il en soit de l'état du monde (et de l'histoire du jeu d'échecs avant moi). En effet, raisonne-t-on, l'intention est un état du sujet, une façon d'être individuelle. On peut donc concevoir que cet état intrinsèque soit réalisé (dans la substance cérébrale ou dans une substance mentale quelconque) pour la première fois dans l'histoire universelle. Cela voudrait dire que quelqu'un aurait, pour la première fois, l'idée qu'il a l'intention de jouer à un jeu qu'il se représente mentalement (et qui est identique à ce que nous appelons les échecs). 
L'argument individualiste peut être poussé plus loin. Il a bien fallu, dira-t-on, que les gens aient un jour l'idée de jouer pour que naisse l'institution. Le jeu d'échecs est une activité de coopération sophistiquée: on n'a pas pu l'inventer machinalement, sans s'en apercevoir. Il s'est donc passé ceci: quelqu'un a eu l'idée de jouer, sinon aux échecs, du moins à quelque chose comme des protoéchecs, et il l'a proposé à quelqu'un d'autre qui l'a accepté. L'institution est alors l'objectivation des conduites individuelles qui, par la répétition, finissent par prendre une forme stable (grâce au succès des précédentes performances).

\section{La socialité de l'état mental}

A cet argument individualiste, Wittgenstein répond que l'intention de faire quelque chose n'est justement pas un état interne d'une personne individuelle, mais que c'est une disposition d'esprit qui n'appartient à cette personne que si certaines conditions contextuelles sont remplies. Notons qu'il ne s'agit pas de poser une condition épistémologique à la description des joueurs, mais une condition logique. Wittgenstein ne dit pas qu'on ne peut pas savoir quelle est l'intention de quelqu'un d'autre si l'on ne connaît pas la situation dans laquelle se trouve le sujet à décrire. Il dit que cela n'a pas de sens de dire que le sujet a l'intention de jouer aux échecs si la condition contextuelle n'est pas satisfaite, pas plus qu'on ne peut dire que la couronne d'or, dans la cérémonie royale, possède intrinsèquement la signification du faste et de la dignité.

Soit un individu dans la situation de notre villageois qui est extérieur à l'institution du jeu d'échecs. Il est logiquement exclu que lui vienne à l'esprit «Je vais jouer aux échecs ». Bien entendu, il peut arriver qu'un individu soit le seul à avoir (maintenant) l'intention de jouer aux échecs. Cela veut dire qu'il propose en vain à divers partenaires éventuels de jouer avec lui. Cet individu a donc son intention dans un contexte où d'autres personnes possèdent déjà l'idée des échecs, savent y jouer ou pourraient apprendre. Par exemple, on peut leur demander: voulez-vous jouer aux échecs? Elles répondent qu'elles n'en ont pas l'intention. Elles pourraient donc avoir cette intention! En revanche, on ne peut pas dire que quelqu'un est actuellement le seul à avoir cette intention au sens parce que l'idée des échecs vient de lui venir, et qu'il est donc le premier.

«Il a l'intention de jouer aux échecs.» En posant le fait psychologique, qui peut être singulier (une seule personne a maintenant cette intention), on pose simultanément les conditions de ce fait (le système qui permet à un individu d'avoir une intention et éventuellement d'avoir une intention qu'il est le seul à avoir). En attribuant cette intention individuelle, nous disons quelque chose de l'esprit de l'agent. Mais nous disons aussi dans quelle sorte de société il vit. Le social est donc présent dans son esprit, mais pas comme un objet de représentation, un objet présent à la conscience ou représenté dans sa personne d'une façon ou d'une autre. Le social est présent à son esprit, dans l'intention même de jouer qui se présente à lui, par le fait de sa participation à la pratique (établie, régulière) du jeu d'échecs.

\section{La socialité de la règle}

On dira ici: mais si c'était vrai, cela voudrait dire que le jeu d'échecs a toujours existé, que les règles ont toujours existé. En effet, l'argument, s'il était 
valide, aurait pour conséquence indésirable d'interdire à un individu d'inventer une règle pour lui-même et d'être le seul à la suivre. A cette objection qu'il se fait à lui-même, Wittgenstein répond ainsi:

\begin{abstract}
Certainement, je peux me donner à moi-même une règle et ensuite la suivre. Mais est-ce cela que je me donne n'est pas une règle uniquement parce que c'est analogue à ce qui s'appelle 'règle' dans le commerce des hommes? (Remarques sur les fondements des mathématiques, VI. 41).
\end{abstract}

Le premier point de cette réponse est qu'il ne doit pas entrer dans l'intention du philosophe de corriger le sens commun, si cela veut dire que le philosophe pourrait, par la force de l'argument philosophique, faire des découvertes renversantes sur nos véritables pouvoirs. En l'occurrence, la philosophie prétendrait nous apprendre qu'en réalité, contrairement à ce que tout le monde a toujours pensé, nous n'avons pas vraiment le pouvoir de nous fabriquer à notre propre usage une nouvelle règle. Il va de soi qu'une telle prétention serait absurde. Bien entendu, une personne particulière peut adopter une règle de conduite dont elle a fixé elle-même le contenu, tout comme elle peut en recevoir une de quelqu'un d'autre.

En réalité, et c'est le second point de la réponse, Wittgenstein est à la recherche d'une condition de sens pour l'usage d'une description de type «Pierre se fixe à lui-même une règle dont il est l'auteur ». On peut certainement dire que Pierre s'est donné à lui-même une règle, mais on le peut à condition de pouvoir dire en quoi ce qu'il s'est donné à lui-même est une règle.

Ce que je me donne à moi-même est-il une règle? En quoi est-ce une règle? Voici une condition pour qu'une règle ait été instituée: la prochaine fois, je ne suis pas libre, les choses sont fixées. Quoi que je fasse à partir de maintenant, ce que je ferai sera en accord avec la règle ou bien y contreviendra. Mais nous devons ici nous demander: comment puis-je faire maintenant, par une initiative mienne, que les choses ne soient plus libres tout à l' heure? Qui va décider, tout à l'heure, si ce que $\mathrm{j}$ 'aurai fait sera ou non en accord avec la règle? Et, comme l'explique Wittgenstein, la réponse à la question de savoir comment c'est bien une règle que je fixe pour moi-même est à chercher dans le sens que prend le mot «règle» entre plusieurs personnes. Que veut dire «règle» dans le «commerce» humain, dans la vie? Le mot veut dire qu'on s'accorde à dire que telle réponse est correcte, telle autre réponse incorrecte. Nous sommes donc renvoyés au consensus, non pas comme à une sorte de contrat social entre Hommes Naturels, mais comme à un arrière-fond présupposé par toute intelligence de la règle. L'homme qui se donne une règle est forcément un Homme Civil.

Quelqu'un peut se fixer une règle à lui-même, mais il ne peut faire cet acte d'autodétermination que dans le contexte social d'une société humaine dans laquelle il y a déjà toutes sortes de règles. Cet individu qui se fixe une règle n'est donc pas un homme à l'état de nature, mais c'est quelqu'un qui met en œuvre des idées qu'il a prises dans la société, des idées sociales. Son invention est donc individuelle au sens descriptif du mot «individu»: elle provient d'un agent que nous savons individuer en indiquant qu'il s'agit d'un individu humain, pourvu d'une biographie humaine (date de naissance, etc.). Mais son invention n'est pas individuelle au sens «normatif» de ce mot d'individu, car l'idée de règle est typiquement une idée sociale, puisque c'est l'idée purement conventionnelle d'une 
différence entre deux comportements possibles d'un agent individuel, l'un correct et l'autre correct. Dire que l'Homme Naturel pourrait se donner une règle à luimême, c'est dire qu'il pourrait passer une convention avec lui-même (plutôt qu'avec nous) et qu'il saurait désormais, grâce à cette convention, ce qu'il doit faire.

Centre de recherches politiques Raymond Aron

Ecole des Hautes Etudes en Sciences Sociales, Paris 